\title{
Methane and carbon monoxide infrared emissions observed at the Canada-France-Hawaii Telescope during the collision of comet SL-9 with Jupiter
}

\author{
J.-P. Maillard ${ }^{1,6}$, P. Drossart ${ }^{2,6}$, B. Bézard ${ }^{2,6}$, C. de Bergh ${ }^{2}$, E. Lellouch ${ }^{2}$, \\ A. Marten ${ }^{2}$, J. Caldwell ${ }^{3,6}$, J.-C. Hilico ${ }^{4}$, and S.K. Atreya ${ }^{5}$
}

\begin{abstract}
Observations with the Fourier Transform Spectrometer were conducted in spectral ranges from 1.6 to $4.7 \mu \mathrm{m}$, from July 17 to 21 (UT) on the hot plumes appearing on the limb as well as hours or days after the impacts. We present here an analysis of the methane emission observed at $3.3 \mu \mathrm{m}$ some $10 \mathrm{~min}$ after the $\mathbf{C}$ impact, indicating the presence of a very small (less than $100 \mathrm{~km}$ wide) hot region with temperatures in the 750-1500 $\mathrm{K}$ range within the $0.1-$ to $0.01-\mathrm{mbar}$ region. We also report the detection of $\mathrm{CO}$ emission at $4.7 \mu \mathrm{m} 4.5 \mathrm{hrs}$ after the $\mathrm{L}$ impact, indicative of a temperature of $274 \pm 10 \mathrm{~K}$ at the $\sim 10^{16} \mathrm{CO}$ molec $\mathrm{cm}^{-2}$ level. The observations suggest that the stratospheric temperature decreases with depth by at least $30 \mathrm{~K}$ over two $\mathrm{CO}$ pressure decades.
\end{abstract}

\section{Observing conditions}

The Fourier Transform Spectrometer (FTS) on the 3.6-m Canada-France-Hawaii Telescope (Mauna Kea, Hawaii) participated in the exceptional campaign for observing the crash of comet Shoemaker-Levy 9 on Jupiter by being scheduled from July 17 to July 21 (UT), 1994. For each observation, a 2.5-arcsec circular aperture, corresponding to a projected diameter of $9400 \mathrm{~km}$ at Jupiter, was centered on an impact site and kept accurately on this position by offset-guiding from a galilean satellite.

Table 1 summarizes the observations which were conducted. Beside a filter centered at $4.7 \mu \mathrm{m}(2080-2180$ $\mathrm{cm}^{-1}$ ) well suited to detect $\mathrm{CO}$, three other filters were

\footnotetext{
${ }^{1}$ Institut d'Astrophysique de Paris, CNRS, France

2 Département de Recherche Spatiale, CNRS-URA264, Observatoire de Paris, Meudon, France

${ }^{3}$ Physics Department, York University, North York, Canada

${ }^{4}$ Laboratoire de Physique de l'Université de Bourgogne, Dijon, France

${ }^{3}$ Department of Atmospheric, Oceanic and Space Sciences, University of Michigan, Ann Arbor, USA

${ }^{6}$ Visiting astronomer at the Canada-France-Hawaii Telescope operated by the National Research Council of Canada, the Centre National de la Recherche Scientifique de France, and the University of Hawaii
}

Copyright 1995 by the American Geophysical Union.

Paper number 95GL01019

0094-8534/95/95GL-01019\$03.00 used, around $3.3 \mu \mathrm{m}\left(2850-3050 \mathrm{~cm}^{-1}\right)$ for $\mathrm{CH}_{4}, 2.3 \mu \mathrm{m}$ (4100-5200 $\left.\mathrm{cm}^{-1}\right)$ and $1.6 \mu \mathrm{m}\left(5400-6800 \mathrm{~cm}^{-1}\right)$. In each filter, spectra of Vega were recorded several hours after the Jupiter observations at similar airmasses to correct for instrumental response and telluric opacity, and to provide absolute flux calibration.

Five sites (B, C, G, L and R) were observed at 4.7 $\mu \mathrm{m}, \mathrm{G}$ being observed twice with a one day interval. CO was detected in emission on the $L$ site (and only there) 4 h30 after impact. Concommitant observations at the United Kingdom Infrared Telescope [Brooke et al., 1994] confirm this detection. It is noteworthy that no CO emission was seen on the G site one day after the impact, probably due to the rapid cooling of the stratosphere. $C$ and $R$ impacts were observed just a few minutes after the explosion, in the 3.3- $\mu \mathrm{m}$ filter. For both impacts, when the plume became visible at Jupiter's limb, a strong emission was seen centered at $\sim 3000 \mathrm{~cm}^{-1}$, which corresponds to the $Q$ branch of the $\nu_{3}$ band of methane. A modelling of this emission is presented here. Methane emissions on impacts $R$ and $W$ were reported at $7.8 \mu \mathrm{m}$ [Sprague et al., 1994; Bjoraker et al., 1994], and, on impact $H$, over a small spectral range near $3.53 \mu \mathrm{m}$, at the edge of the range observed with the FTS [Encrenaz et al., this issue].

\section{$\mathrm{CH}_{4}$ emission at impact $\mathrm{C}$}

Spectra of the 3.3- $\mu \mathrm{m} \mathrm{CH}_{4}$ emission were recorded with an integration time of 5 min for impact C. To enhance the signal-to-noise ratio, the spectral resolution was degraded to $1.7 \mathrm{~cm}^{-1}$, corresponding to an effective integration time of $38 \mathrm{sec}$. Figure 1 shows the spectrum at impact $\mathrm{C}$, which exhibits the strongest emission feature at $3001 \mathrm{~cm}^{-1}$. Similar spectra were obtained for impact $R$, fainter than on impact $C$. We focus here on the impact $C$ spectrum. A good fit with pure methane emission is obtained only when re-absorption of hot methane emission is allowed in the cold atmosphere of Jupiter. The timing of the observation (maximum emission on 17:07:20 UT), compared to the geometry of the impacts on Jupiter [Chodas and Yeomans, 1994] implies that the observations occured at very large emission angles. An emission angle of $90^{\circ}$ (known only to $5^{\circ}$ because of the uncertainty on the impact time) is assumed in the model, with full account of the spherical geometry in the radiative transfer. 
Table 1. FTS OBSERVATIONS OF SL9/JUPITER

\begin{tabular}{lcccc}
\hline Frag. & \multicolumn{2}{c}{ Observation $^{a}$} & Range $\left(\mathrm{cm}^{-1}\right)$ & Resolution \\
\hline B & $17: 04: 58$ & $05: 48$ & $2080-2180$ & $2.5 \times 10^{4}$ \\
C $^{b}$ & $17: 06: 53$ & $07: 52$ & $2850-3050$ & $1.4 \times 10^{4}$ \\
& $17: 07: 59$ & $08: 51$ & $2080-2180$ & $2.5 \times 10^{4}$ \\
E & $20: 06: 31$ & $06: 50$ & $5400-6800$ & $2.1 \times 10^{4}$ \\
G & $19: 06: 39$ & $07: 11$ & $2080-2180$ & $2.5 \times 10^{4}$ \\
& $19: 07: 28$ & $07: 55$ & $4100-5200$ & $1.5 \times 10^{3}$ \\
& $20: 03: 21$ & $04: 22$ & $2080-2180$ & $2.5 \times 10^{4}$ \\
H & $19: 08: 01$ & $08: 50$ & $4100-5200$ & $1.5 \times 10^{3}$ \\
K & $21: 06: 5307: 57$ & $5400-6800$ & $2.1 \times 10^{4}$ \\
L & $20: 02: 44$ & $03: 16$ & $2080-2180$ & $2.5 \times 10^{4}$ \\
R & $21: 05: 36$ & $05: 52$ & $2850-3050$ & $1.0 \times 10^{4}$ \\
& $21: 06: 33$ & $06: 48$ & $2080-2180$ & $2.5 \times 10^{4}$ \\
& $21: 07: 04$ & $07: 26$ & $5400-6800$ & $2.1 \times 10^{4}$ \\
\hline
\end{tabular}

\footnotetext{
astart dd:hh:mn end hh:mn (UT)

bobserved in real time; max of $\mathrm{CH}_{4}$ emission at 17:07:20

observed in real time; max of $\mathrm{CH}_{4}$ emission at 21:05:44
}

The hot emission is assumed to originate from an isothermal volume, at temperature $T$, located at a mean pressure $P$, with a vertical extension $H$ and an horizontal extension $D$. $H$ is assumed to be comparable to the unperturbed atmospheric scale height, i.e. about $25 \mathrm{~km}$ in the high stratosphere. Since the intensity of the emission strongly depends on temperature, this is equivalent to assuming that a region having the maximum temperature over one scale height altitude range mainly contributes to the observed emission. The free parameters of the model are therefore $D, T$ and $P$. Outside the hot region, the atmosphere is supposed to keep its nominal temperature profile, taken from [Gladstone and Skinner, 1989], which at high altitudes is nearly isothermal with a temperature around $170 \mathrm{~K}$. The methane vertical profile is taken from the same reference, with a tropospheric abundance around $0.2 \%$. In the hot region, the methane mixing ratio has been decreased by a factor of two to roughly take into account the mixing of the cometary, methane-free material, with the atmosphere of Jupiter. Calculations were performed for pressure levels $P$ between $1 \mathrm{mbar}$ and $1 \mu$ bar and temperatures between 500 to $2000 \mathrm{~K}$. Jupiter's atmosphere is very transparent at these altitudes, most of the haze absorbing at higher pressures, and gaseous opacity of methane must be the dominant absorption in this range. Other hydrocarbons have absorption bands near $3.3 \mu \mathrm{m}$, but their column density is $10^{-4}$ to $10^{-6}$ that of methane. Therefore, calculations with only methane lines were performed, including high $J$ values (up to $J=25$ ) as well as the hot bands of $\mathrm{CH}_{4}$ present in the range. More than 40,000 lines were included from a theoretical model by [Hilico et al., 1994]. In addition to the methane lines, a small continuum was added, which may describe the emission of the heated dust in the fireball. This continuum is added mainly for consistency with the model used in [Encrenaz et al., this issue], where such a continuum was found to be present.
The strong telluric methane absorptions present in the spectral range complicate the comparison between synthetic and observed spectra. Thanks to the Doppler shift of Jupiter $\left(0.169 \mathrm{~cm}^{-1}\right.$ in the spectrum of Fig. 1), the Jovian emission is still observable from the ground, but strongly perturbed, even at medium spectral resolution. Since division by a star spectrum at high resolution is not possible, because of the large number of strongly saturated lines in both spectra, the data are instead compared by multiplying the synthetic spectrum (shifted by the Doppler shift of Jupiter) by the star spectrum at high spectral resolution, and then by convolving the resulting spectrum to the resolution of the Jupiter spectrum. The normalization is obtained from the magnitude of the star, and allows us to convert the synthetic spectrum into the original instrumental units (Fig. 1).

A fit to the spectra is found for pressure levels $10^{-5}<$ $P<10^{-4}$ bar and temperatures $750<T<1500 \mathrm{~K}$. The influence of pressure and temperature variations is different on the spectral shape in the range 2850 $2950 \mathrm{~cm}^{-1}$ ( $P$ branch), $3000 \mathrm{~cm}^{-1}$ peak and $3008 \mathrm{~cm}^{-1}$ secondary peak, giving the above constraints on $P$ and $T$. Then, the absolute flux calibration from the Vega spectra allows an estimate of the size of the emitting
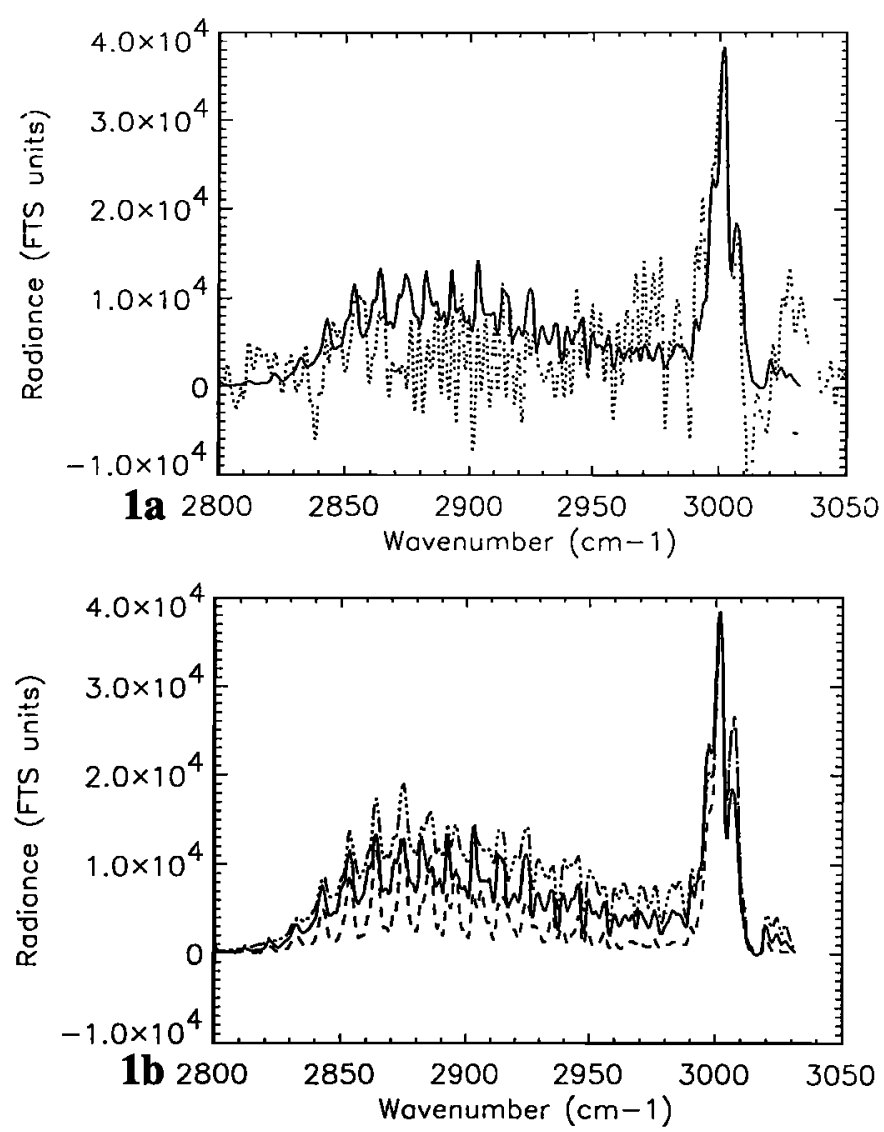

Figure 1. Spectrum of impact $C$ on July 17:07:20 $\mathrm{UT}$, at a resolution of 1700 (dotted line); the $\mathrm{S} / \mathrm{N}$ ratio is about 10; the intensity is in original instrumental counts. The solid line is the best fitting model, with parameters: $P=10^{-4}$ bar, $T=1000 \mathrm{~K}, D=50 \mathrm{~km}$; the peak spectral radiance near $3002 \mathrm{~cm}^{-1}$ is about $5 \times 10^{-9} \mathrm{~W}$ $\mathrm{cm}^{-2} \mathrm{sr}^{-1} / \mathrm{cm}^{-1}$. 
region. The vertical size being assumed to be roughly $25 \mathrm{~km}$, the horizontal dimension is found to lie between $20 \mathrm{~km}$ (for the highest temperature) and $100 \mathrm{~km}$ (for the lowest temperature). The emitting area is therefore very small compared to the aperture ( $9400 \mathrm{~km}$ wide). Nevertheless, it must be emphasized that these results rely on several assumptions. Improvements will come from a comparison with data from other observations

\section{CO emission at Impact $L$}

The 4.7- $\mu \mathrm{m}$ spectrum recorded on the $L$ site, 4.5 hrs after the impact, consists of two components: thermal emission originating from Jupiter's troposphere at pressure levels of a few bars, and $\mathrm{CO}$ emission from the stratosphere locally heated by the impact (Fig. 2). Twelve individual lines from the (1-0) band of ${ }^{12} \mathrm{CO}$ are detected above the $3 \sigma$ level (P2, P4, P7-P12, R2, R3, R5, R6). The weighted-averaged intensity of the P1-P12 and R1-R7 lines is found to be $0.113 \pm 0.006$ erg $\mathrm{s}^{-1} \mathrm{~cm}^{-2} \mathrm{sr}^{-1} / \mathrm{cm}^{-1}$ with an additional $20 \%$ uncertainty from the flux calibration. The corresponding brightness temperature is $222 \pm 3 \mathrm{~K}$. Individual lines from the ${ }^{13} \mathrm{C}$ isotope are within the noise. However, averaging the observed radiances for the nine most intense lines in the bandpass (R2-R7, R9-R11) yields a mean line intensity of $0.019 \pm 0.008 \mathrm{erg} \mathrm{s}^{-1} \mathrm{~cm}^{-2} \mathrm{sr}^{-1} / \mathrm{cm}^{-1}$, indicating a marginal detection of an emission from ${ }^{13} \mathrm{CO}$ at the 2.5- $\sigma$ confidence level.
While the thermal background from the deep troposphere covers the 2.5-arcsec field of view, $\mathrm{CO}$ emission is limited to the area of the $L$ site. To analyze these data, we assumed that the $L$ impact site filled half the area of the field of view at the time of the observations. This would correspond to a $14000-\mathrm{km}$ broad region on Jupiter's disk (7000-km in the projection), consistent with $10-\mu \mathrm{m}$ images of the $\mathrm{L}$ site, $2 \mathrm{hr}$ after the collision [Lagage et al., 1994].

Observations were modelled by means of standard radiative transfer calculations. The temperature model outside of the impact site is taken from [Griffith et al., 1992]. It pertains to cold regions of the STZ. Over the $\mathrm{L}$ site, two modifications were brought to this model above the 0.3-mbar level: the temperature was increased above its nominal $\approx 170 \mathrm{~K}$ value as a consequence of energy deposition from the impact, and the CO mixing ratio was increased above its tropospheric value of $\approx 1 \times 10^{-9}$. In a first step, we used the $C O$ mixing ratio profile $\left(4 \times 10^{-5}\right.$ at pressure levels higher than 0.3 mbar) derived by [Lellouch et al., 1994] from millimeter observations of the G impact site, approximately 10 hours after impact. The lines are then optically thick and emission from ${ }^{12} \mathrm{CO}$ is formed near the $\sim 10^{16} \mathrm{CO}$ molec $\mathrm{cm}^{-2}$ level ( $\mathrm{p} \sim 2 \mu \mathrm{bar}$ ). ${ }^{13} \mathrm{CO}$ emission originates from $\sim 100$ times denser levels, assuming a terrestrial ${ }^{12} \mathrm{C} /{ }^{13} \mathrm{C}$ ratio. The intensities of the ${ }^{12} \mathrm{CO}$ lines can be reproduced adopting a uniform temperature of $267 \mathrm{~K}$ above the 0.3-mbar level. However, the model would then predict intensities for the R2-R12

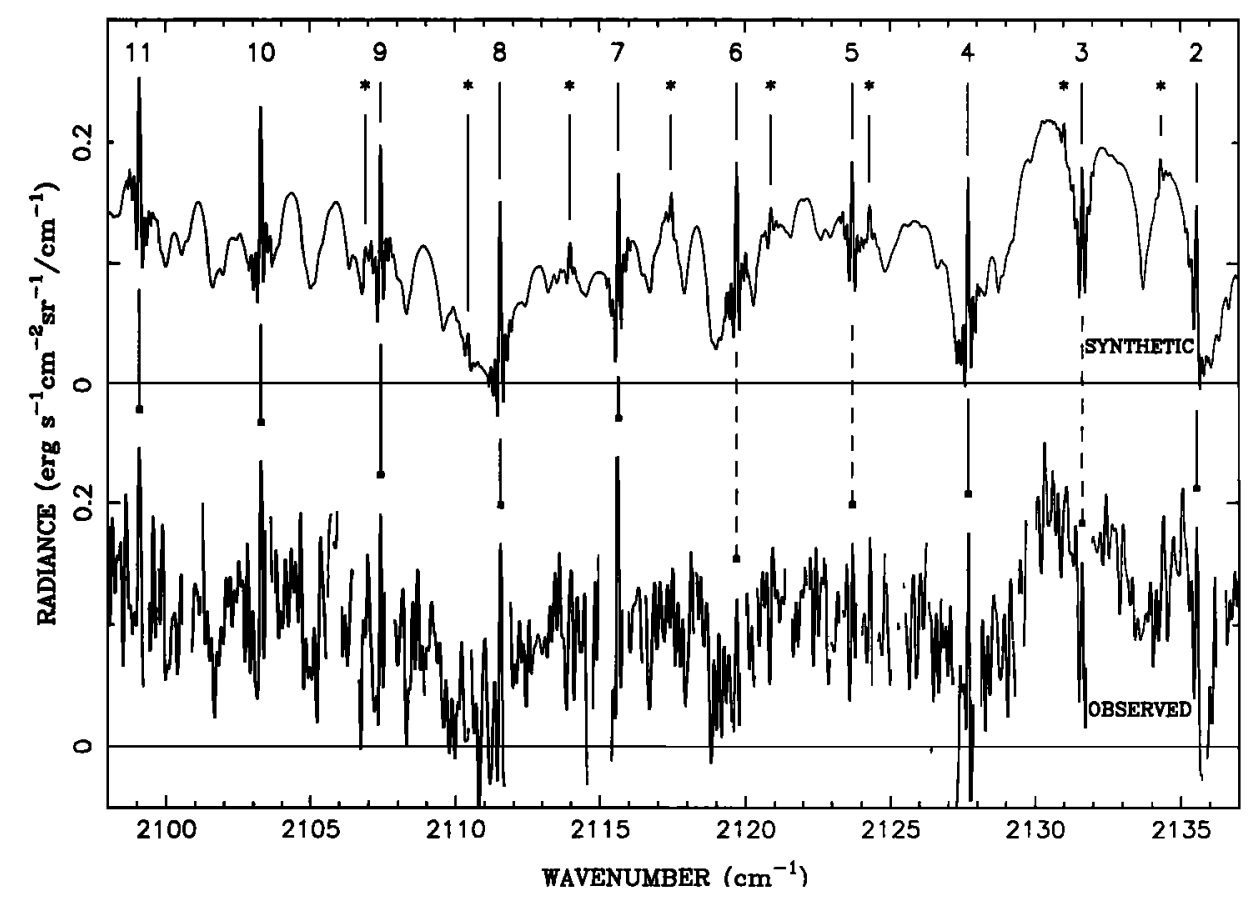

Figure 2. Portion of the 4.7- $\mu \mathrm{m}$ spectrum of the $\mathrm{L}$ site recorded 4.5 hrs after the impact at a resolution of $0.08 \mathrm{~cm}^{-1}$. Data corresponding to regions where the telluric transmission is less than 0.50 are not plotted. The $1 \sigma$ noise level varies between 0.02 and 0.04 erg $\mathrm{s}^{-1} \mathrm{~cm}^{-2} \mathrm{sr}^{-1} / \mathrm{cm}^{-1}$. Many emission lines from the $\mathrm{P}-$ branch of the (1-0) band of ${ }^{12} \mathrm{CO}$, marked with their rotational number, are detected with a $\mathrm{S} / \mathrm{N}$ ratio higher than 3 (solid lines). Lines from the ${ }^{13} \mathrm{CO}$ isotope (indicated as $*$ ) are below the $3 \sigma$ level. The best fit synthetic spectrum, assuming an isothermal CO layer at $274 \mathrm{~K}$ (see text), is shown for comparison. 
${ }^{13} \mathrm{CO}$ lines about half those of the ${ }^{12} \mathrm{CO}$ lines, in conflict with the observations. This conflict can be resolved in two ways: either by decreasing the $\mathrm{CO}$ column density to reduce the line optical depths, or by assuming a temperature gradient within the region where $\mathrm{CO}$ is present.

In the first hypothesis, where an isothermal profile is adopted within the plume, we find that a CO column density equal to $1.5 \pm 0.8 \times 10^{17}$ molec $\mathrm{cm}^{-2}$ is needed to simultaneously reproduce the ${ }^{12} \mathrm{CO}$ and ${ }^{13} \mathrm{CO}$ emission. The corresponding mass of $\mathrm{CO}$ is then $1.0 \pm 0.5 \times 10^{13} \mathrm{~g}$, a factor of 5-15 less than inferred by [Lellouch et al., 1994] for the $G$ impact. In this model, the temperature of the plume is $274 \pm 10 \mathrm{~K}$, taking into account the noise level, uncertainties on the flux calibration (20\%) and on the dilution factor of the impact site in the aperture $(+50 \% /-25 \%)$. This best fitting model is displayed in Fig. 2.

In the second class of models, we assumed that the CO column density was that given by Lellouch et al. $\left(1.4 \times 10^{18}\right.$ molec $\left.\mathrm{cm}^{-2}\right)$. Because the ${ }^{12} \mathrm{CO}$ emission originates higher than the ${ }^{13} \mathrm{CO}$ emission, a positive lapse rate ( $\mathrm{dT} / \mathrm{dlogp}>0$ and assumed to be constant) allows us to reduce the intensities of the ${ }^{13} \mathrm{CO}$ lines with respect to the ${ }^{12} \mathrm{CO}$ lines. In this case, we derive a temperature difference of $37 \pm 8 \mathrm{~K}$ between the $10^{16}$ and $10^{18} \mathrm{CO}$ molec $\mathrm{cm}^{-2}$ levels, i.e. over $4.5 \mathrm{CO}$ density scale heights. The error bars correspond to the $1 \mathrm{SD}$ noise uncertainty on the ${ }^{13} \mathrm{CO}$ lines. More conservatively, at the $3-\sigma$ level, we conclude that this temperature difference is at least $30 \mathrm{~K}$. The temperature at the upper level is still $274 \pm 10 \mathrm{~K}$, taking into account all uncertainties. If $\mathrm{CO}$ is uniformly mixed with gas above the 0.3-mbar level, the above atmospheric levels correspond to pressures of 2 and $200 \mu$ bar.

Since the present observations and those reported in Lellouch et al. refer to different impact sites ( $L$ and G) and different elapsed times since impact (4h30 and 10h), it is perhaps not surprising that the corresponding solutions are, at face value, different. Nevertheless, it is worth noting that a consistent solution for the two datasets can be found. Fitting the millimeter data with the thermal profile retrieved for our second class of models ( $T=274 \mathrm{~K}$ near $2 \mu$ bar and $\mathrm{T}=237 \mathrm{~K}$ near $200 \mu$ bar) leads to a column density of about $8 \times$ $10^{27}$ molec $\mathrm{cm}^{-2}$, i.e. 1.7 time less than in Lellouch et al.'s original analysis and in our modelling. This CO abundance and a profile warmer than given above by a few degrees would then satisfy both sets of observations. We defer a detailed joint analysis of millimeter and infrared observations to future work.

\section{References}

Bjoraker, G.L., Herter, T., Gull, G., Stolovy, S., and B. Pirger, Detection of water in the fireball of fragments $G$ and $\mathrm{K}$ of comet Shoemaker-Levy 9, Bull. Amer. Astron. Soc., 26, 1578, 1994.

Brooke, T.Y., Orton, G.S., Crisp, D., Friedson, A.J., and G. Bjoraker, Near-infrared spectroscopy of the ShoemakerLevy 9 impact sites with UKIRT: $\mathrm{CO}, \mathrm{NH}_{3}$, and hase layers, Bull. Amer. Astron. Soc., 26, 1585, 1994.

Chodas, P.W., and D.K. Yeomans, Comet Shoemaker-Levy 9 impact times and impact geometries, Bull. Amer. Astron. Soc., 26, $1569,1994$.

Encrenaz, Th., R. Schuls, J. A. Stūwe, G. Wiedemann, P. Drossart, and J. Crovisier, Near-IR spectroscopy of Jupiter at the time of SL9 impact: emissions of $\mathrm{CH}_{4}, \mathrm{H}_{3}^{+}$ and $\mathrm{H}_{2}$, Geophys. Res. Lett., in press, 1994.

Gladstone, G. R., and T. E. Skinner, Spectral Analysis of Jovian auroral emissions, in Time-Variable phenomena in the Jovian system, NASA-SP 494, pp. 221-228, M.J.S. Belton, R.A. West and J. Rahe Eds, Washington DC, 1989.

Griffith, C.A., Bézard, B., Owen, T., and D. Gautier, The tropospheric abundances of $\mathrm{NH}_{3}$ and $\mathrm{PH}_{3}$ in Jupiter's Great Red Spot from Voyager IRIS observations, Icarus, 98, 82-93, 1992.

Hilico, J.C., Champion, J.P., Toumi. S., Tyuterev, Vl.G., and S.A. Tashkun, New analysis of the pentad system of methane and prediction of the (pentad-pentad) spectrum, J. Mol. Spectrosc., 168, 455-476, 1994.

Lagage, P.O., et al., $10 \mu \mathrm{m}$ observations of SL9 impacts with Camiras at NOT, Bull. Amer. Astron. Soc., 26, 1586, 1994.

Lellouch, E. et al., Shock chemistry in Jupiter's atmosphere following the impact of comet Shoemaker-Levy 9, Nature, in press, 1995.

Sprague, A.L., et al., KAO observations of Jupiter during and following the impact of comet SL-9 fragments $R$ and W using HIFOGS (4.9-9.4 and 9.3-14.5 $\mu \mathrm{m})$, Bull. Amer. Astron. Soc., 26, 1579, 1994.

Sushil K. Atreya, Department of Atmospheric, Oceanic and Space Sciences, Space Research Building, University of Michigan, Ann Arbor, Michigan 48109-2143, USA

Bruno Bézard, Catherine de Bergh, Pierre Drossart, Emmanuel Lellouch, André Marten, Département de Recherche Spatiale, CNRS-URA 264, Observatoire de Paris, Section de Meudon, 92195 Meudon cedex, France John Caldwell, Physics Department, York University, 4700 Keele Street, North York, Ontario M3J1P3, Canada Jean-Claude Hilico, Laboratoire de Physique de l'Universite de Bourgogne, 6 Boulevard Gabriel, 21000 Dijon, France

Jean-Pierre Maillard, CNRS, Institut d'Astrophysique de Paris, 98 ${ }^{\text {bis }}$ Boulevard Arago, 75014 Paris, France

(received December 7, 1994; revised February 2, 1995; accepted March 10, 1995.) 